\title{
Levantamento epidemiológico sobre fraturas de osso frontal atendidas pelo serviço de residência em cirurgia buco-maxilo da Universidade Estadual de Maringá, entre 2009 a 2012.
}

\author{
Fourth years retrospective evaluation of fractures of the frontal bone in the residency \\ program in oral and maxillofacial surgery, state university of maringa.
}

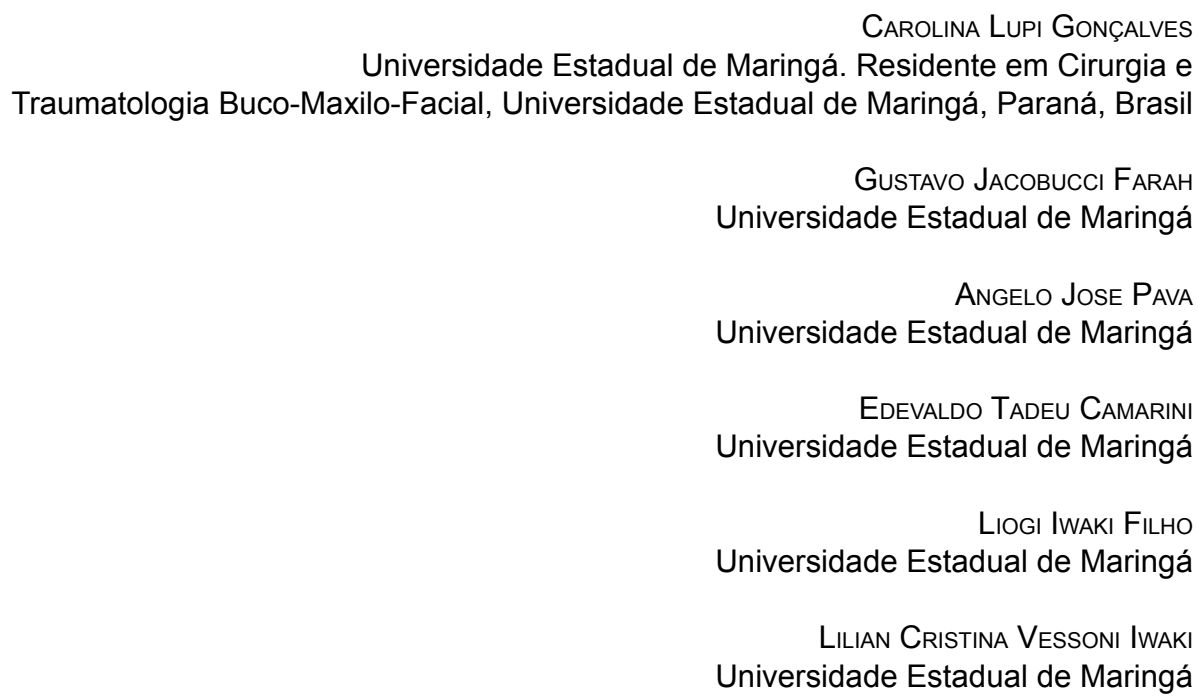

\section{RESUMO:}

Objetivo: realizar estudo epidemiológico retrospectivo através da avaliação de prontuários de pacientes diagnosticados com fratura de osso frontal entre 2009 a 2012 e confrontar seus dados com a literatura recente. Métodos: avaliação de 51 prontuários de pacientes diagnosticados com fratura de osso frontal e levantamento dos dados demográficos, causas, tipos de fratura, meios de diagnóstico e tratamento realizados e tabulação dos dados. Resultados: Foram avaliados 51 pacientes com fratura frontal associada a outras fraturas, sendo $94,11 \%$ do gênero masculino e . A idade média foi de 34,39 anos; 48 pacientes do gênero masculino (94,11\%). A causa prevalente foi acidente de moto em $39,21 \%$, seguido de automobilístico, com $13,75 \%$. O diagnóstico foi realizado pela tomografia computadorizada e houve prevalência de fratura da parede anterior do seio frontal em $74,5 \%$ dos casos, acompanhada de fratura nasal $(35,9 \%)$ e fratura tipo NOE $(13,72 \%)$. O tratamento foi conservador em todos os casos. Conclusão: As fraturas de osso frontal correspoderam a cerca de 5,37\% desta casuística, geralmente associadas a outras fraturas, principalmente nasal e NOE. Foram prevalentes nos pacientes do gênero masculino, tendo como principal causa os acidentes de moto. A idade média foi de 34,39 anos. O diagnóstico foi realizado por tomografia computadorizada , encontrando-se prevalência de fratura na parede anterior do seio frontal e a principal consequência foi rinorreia, nos pacientes com fratura na parede posterior do seio frontal. $O$ tratamento preconizado foi o cirúrgico conservador.

Palavras-chave: osso frontal, levantamento epidemiológico, cirurgia bucal. 
LEVANTAMENTO EPIDEMIOLÓGICO SOBRE FRATURAS DE OSSO FRONTAL ATENDIDAS PELO SERVIÇO DE

RESIDÊNCIA EM CIRURGIA BUCO-MAXILO dA UnIVERSIDAde EstaduAl de MARINGÁ, ENTRE 2009 a 2012.

\begin{abstract}
Objective: Retrospective evaluation of patients diagnosed with fracture of the frontal bone between 20092012 and to compare their data with the recent literature. Methods: Review of medical records of 51 patients diagnosed with a fracture of the frontal bone and data of health survey, etiology, fracture types, diagnosis and treatment in this patients. Results: 51 patients were evaluated with frontal fracture associated with other fractures, with $94.11 \%$ of males and. The average age was 34.39 years; 48 male patients $(94.11 \%)$. The prevalent cause was motorcycle accident at $39.21 \%$, followed by automobile, with $13.75 \%$. The diagnosis was made by CT scan and there was prevalence of fracture of the anterior wall of the frontal sinus in $74.5 \%$ of cases, accompanied by nasal fracture (35.9\%) and NOE fracture type (13.72\%). The conservative treatment was in all cases. Conclusion: Fractures of the frontal bone result approximately $5.37 \%$ of this sample, usually associated with other fractures, especially nasal and NOE. It was prevalent in male patients, the main cause motorcycle accidents. The average age was 34.39 years. The diagnosis was made by computed tomography, lying prevalence of fractures in the anterior wall of the frontal sinus and the main consequence was rhinorrhea in patients with fracture of the posterior wall of the frontal sinus. The patient was treated with conservative surgery.
\end{abstract}

Keywords: Frontal bone, health surveys, oral surgery.

\section{INTRODUÇÃO}

As fraturas do seio frontal correspondem em média de $5 \%$ a $12 \%$ das fraturas em face $^{1,2,3,4,5}$. Assim como qualquer tipo de fratura tem etiologia conhecida e bem definida: acidentes automobilísticos ${ }^{1,2,6}$, acidentes motociclísticos, agressões físicas, dentre outros $^{2,3,4}$. Embriologicamente, o osso frontal é do tipo intramembranoso e a ossificação ocorre entre a $8^{0}$ e $9^{0}$ semana de gestação. A primeira etapa da pneumatização ocorre no $4^{0}$ mês após o nascimento e se estende até por volta dos $2 \operatorname{anos}^{4,7}$. A pneumatização completa do seio frontal termina por volta dos 12 aos 16 anos e os septos ósseos apresentam configuração extremamente variável. $\mathrm{O}$ seio frontal é ausente em 4\% da população ${ }^{2,5,7}$ e apresenta superfície aproximada de $720 \mathrm{~mm}^{2,7}$. A mucosa do seio frontal é do tipo respiratória com epitélio ciliado de 0,7 a $2 \mathrm{~mm}$ de espessura, a velocidade ciliar é de cerca de 250 ciclolminuto e é mais rápida ao redor do ducto nasofrontal. A camada de mucina flui de modo espiral, de lateral para medial sendo mais lento no teto do seio frontal ${ }^{4}$. A área óssea mais delgada é a região da glabela enquanto que regiões como arcos superciliares são mais espessas ${ }^{7}$.

A maioria dos pacientes diagnosticados com fratura de osso frontal apresenta outros tipos de fratura de face associados. Fraturas nasal, tipo NOE e do complexo orbitozigomático-maxilar, por conta da proximidade anatômica, estão entre as mais presentes ${ }^{1,5,7}$. A classificação das fraturas de osso frontal é vasta e controversa. Para o tratamento a verificação da permanência do ducto nasofrontal é determinante a fim de se minimizar possíveis complicações pós-operatórias. A necessidade de obliteração ou cranialização do seio frontal depende do envolvimento da parede anterior do seio frontal, ducto nasofrontal e parede posterior do seio frontal ${ }^{1}$.

Neste trabalho realizou-se um estudo epidemiológico retrospectivo através de avaliação de prontuários pertencentes ao serviço de Residência em Cirurgia e Traumatologia Buco-Maxilo-Facial da Universidade Estadual de Maringá, Maringá, Paraná, Brasil- entre os anos de 2009 a 2012. As informações revisadas incluíram idade e gênero, etiologia do trauma, fraturas associadas, tipo de tratamento e complicações e ainda foram tecidas considerações sobre diagnóstico, classificações e possibilidades de tratamento.

\section{Material e Métodos}

Foram revisados 948 prontuários pertencentes ao serviço de Residência em Cirurgia e Traumatologia Buco-Maxilo-Facial da Universidade Estadual de Maringá, Maringá, 
Paraná, Brasil, entre os anos de 2009 a 2012. Destes, 51 prontuários eram referentes à pacientes diagnosticados com fratura do osso frontal associada ou não a outras fraturas em face. As informações revisadas incluíram idade e gênero, etiologia do trauma, fraturas associadas, tipo de tratamento e complicações, dentre outras. Todos os pacientes participantes da pesquisa não foram identificados e autorizaram previamente o uso de seus dados para pesquisas neste Serviço. O Departamento de Odontologia, ao qual a residência em Cirurgia e Traumatologia BucoMaxilo-Facial é vinculada, onde esta pesquisa foi realizada, também autorizou e é ciente do levantamento de dados dos prontuários.

\section{Resultados}

No período de 2009 a 2012, foram atendidos 51 pacientes diagnosticados com fratura osso frontal, por vezes associada a outras fraturas em face. A média de idade foi de 34,39 anos; 48 pacientes do gênero masculino $(94,11 \%)$ e 3 pacientes do gênero feminino $(11,76 \%)$. A etiologia do trauma encontrada foi bastante variada: acidente motociclístico (20 casos- $39,21 \%$ ) foi o mais prevalente, seguido de acidente automobilístico (7 casos-13,75\%) e acidente ciclístico (6 casos$11,76 \%$ ), acidente de trabalho (5 casos- $9,8 \%$ ), agressão física (4 casos- 7,8\%), queda de nível e acidente esportivo ( 2 casos cada$3,91 \%)$, projétil de arma de fogo em face (1 caso- 1,95\%), atropelamento (1 caso- 1,96\%) e em três casos a etiologia era desconhecida (5,88\%). Dos 51 pacientes, 15 apresentaram fratura isolada de seio frontal $(29,41 \%)$, em 38 casos houve fratura isolada da parede anterior do seio frontal $(74,50 \%)$ e em 13 casos ambas as tábuas estavam fraturadas ( $25,49 \%)$. Dos pacientes diagnosticados com fratura da parede posterior do seio frontal, $9(69,23 \%)$ apresentaram rinorréia e em todos os casos o tratamento foi conservador.
As fraturas do seio frontal normalmente estavam associadas a vários outros tipos de fraturas face e crânio. A fratura nasal foi a mais comumente encontrada: 18 casos (35,29\%). A seguir, observou-se fratura do tipo NOE em 7 casos $(13,72 \%)$, fratura de zigoma em 6 casos $(11,76 \%)$,fratura Le Fort II em 5 casos $(9,08 \%)$, fratura de órbita em 4 casos $(7,84 \%)$, fratura de temporal em 3 casos $(5,88 \%)$, e ainda fraturas Le Fort I, Le Fort III e mandíbula, lanelong foram encontradas em 2 casos cada (3,92\%). Ainda, fratura de parietal foi encontrada somente em 1 caso $(, 96 \%)$. De todos pacientes atendidos, 32 foram tratados conservadoramente e em 19 casos houve intervenção cirúrgica. Em dois casos, pacientes evoluiram para ptose da pálpebra superior direita e somente em um caso de fratura de osso frontal associada à fratura nasal o paciente evoluiu com discreta redução da permeabilidade nasal esquerda. Dos pacientes tratados cirurgicamente, o ducto nasofrontal encontrava-se obstruído em 1 caso, sendo necessária a obliteração deste no momento da cirurgia. Em todos os casos de pacientes com fratura da parede posterior do seio frontal, a equipe de Neurocirurgia responsável pelos atos, tratouos conservadoramente, não havendo portando, nenhum caso de cranialização do seio frontal entre estes pacientes.

\section{Discussão}

As fraturas de seio frontal acontecem de $5 \%$ a $15 \%$ das fraturas em face ${ }^{3}$, e possuem etiologia diversa. São raras em crianças e acometem principalmente pacientes do gênero masculino entre os 20 e 30 anos $^{4,7,8,9}$. Forças impactantes diretamente sobre a região anterior do crânio ocasionam este e outros tipos de fraturas; projéteis por arma de fogo e acidentes de trabalho costumam causar comunicação óssea por se tratar de grande quantidade de força aplicada em pequena área². Observamos que dos 948 prontuários 
revisados, 51 apontavam pacientes com fratura de osso frontal. Destes, 48 eram pertencentes a pacientes do gênero masculino $(94,11 \%)$ e 3 a pacientes do gênero feminino; a idade variou de 18 a 57 anos com média de 34,39 anos, em concordância com demais autores.

A literatura observa em relação à vasta etiologia do trauma que acidentes automobilísticos são mais prevalentes, seguidos de agressões físicas e acidentes motociclísticos ${ }^{3}$, no entanto em nesta experiência encontramos acidentes motociclísticos $(39,21 \%)$ como mais comuns, seguido de acidentes automobilísticos $(13,72 \%)$ e acidentes ciclísticos (11,76\%), acidentes de trabalhos $(9,8 \%)$, agressões físicas $(7,84 \%)$, queda de nível e acidentes esportivos $(3,92 \%$ cada), projétil de arma de fogo em face $(1,96 \%)$, atropelamento $(1,96 \%)$ e em três casos a etiologia era desconhecida $(5,88 \%)$. Parece razoável que o fato de veículos motomotores serem muito comum como meio de transporte nesta região, há maior risco de traumas que resultem neste tipo de fratura.

$A$ associação às demais fraturas em face e crânio - fraturas NOE, zigoma-maxila - são frequentemente relatadas na literatura ${ }^{3,4}$ - Sivori ${ }^{1}$ relata em uma revisão de 43 prontuários odontológicos entre 2000 a 2006, que juntamente com fraturas de seio frontal estão entre as mais presentes: NOE $(39,5 \%)$, complexo zigomático-maxilar $(37,2 \%)$, órbita $(34,9 \%)$, Le Fort I $(18,6 \%)$, mandíbula (14\%), nasal $(11,6 \%)$, Le Fort II (7\%) e ainda lanelong, Le Fort III e temporal (2,3\%). Neste estudo mostrou-se muito semelhante, excetuandose o fato de que fraturas nasais foram mais prevalentescom $35,29 \%$ dos casos, seguidas de fraturas NOE $(13,72 \%)$, complexo zigomáticomaxilar $(11,76 \%)$, Le Fort II em 5 casos $(9,08 \%)$, órbita $(7,84 \%)$, fratura de temporal em 3 casos ( $5,88 \%$ ), e ainda fraturas Le Fort I, Le Fort III, mandíbula, lanelong em 2 casos cada ( $3,92 \%)$ e parietal $(1,96 \%)$. É Importante salientar que assim como na literatura, clinicamente pacientes diagnosticados com fratura de osso frontal apresentavam edema local, comumente parestesia do nervo supraorbitário do lado acometido, equimose local, alterações estéticas por depressão do osso frontal, rinorréria (9 casos) e limitação de movimentos oculares (2 casos) ${ }^{4,5}$. A tomografia computadorizada é o padrão ouro para confirmação do diagnóstico, observação da extensão da fratura e planejamento cirúrgico ${ }^{1,3,4,5,6,7}$; através dos cortes coronais pode-se observar fraturas do soalho do seio frontal e teto de órbita e pelos cortes sagitais o ducto nasofrontal ${ }^{4}$, embora Miloro et al. (2009) observe que através da tomografia computadorizada a confiabilidade na detecção do ducto é escassa.

Existem vários tipos de classificação para fraturas de seio frontal. Segundo Miloro (2009), são esquemas complexos, sem valor e que complicam o processo diagnóstico; assim, deveriam ser realizadas somente considerações sobre tábua óssea anterior, tábua óssea posterior, ductos nasofrontais, lesões intracranianas e demais fraturas da face. Para Bell(2009), os seios paranasais, cavidade nasal e cavidade orbitária são considerados absorvedores de forças, e assim, as fraturas são divididas em dois grandes grupos: lesões tipo I e tipo II. As lesões tipo I consistem em fraturas fronto-naso-etmoidais e medial de órbita, sem base do crânio; caracterizam-se por absorver e neutralizar o impacto, preservando a parede posterior do seio frontal, a base do crânio e o nervo óptico. Não apresentam lesão cerebral significativa ou laceração de duramáter. Já as lesões tipo II envolvem base do crânio, suturas fronto-naso-etmoidais e parede medial de órbita por vezes com compressão do nervo óptico. Caracterizam-se por traumas de alta energia que não são absorvidas e resultam em graves rupturas de várias regiões da face e crânio. Ocorre frequentemente deslocamento intracraniano da parede 
posterior do seio frontal, afundamento da pirâmide nasal e deslocamentos intracranianos ao longo da base de crânio, incluindo o teto orbital e seio esfenoidal. Lesões neurológicas importantes são comuns: lesões durais, rinorréia, hérnia de tecido cerebral, hematoma intracraniano e compressão do nervo óptico. No entanto, Doonquah, Brown e Mullings (2012) entendem que as fraturas podem ser classificadas em 5 tipos, a saber: Tipo 1 (fratura da parede anterior com o mínimo cominuição, não há NOE ou fratura de órbita associada), Tipo 2 (parede anterior cominuta com possível NOE e / ou fratura de órbita), Tipo 3 (fraturas da parede anterior e posterior , sem deslocamento significativa da parede posterior), Tipo 4 (fraturas da parede anterior e posterior com lesão dura-máter e perda de líquido cefalorraquidiano), Tipo 5 (fraturas da parede anterior e posterior com lesão dural, vazamento de fluido cérebro-espinhal, perda de tecido duro e/ou mole e grave ruptura da fossa craniana anterior).

Assim como no tratamento das demais fraturas, a redução aberta tem como objetivo a proteção das estruturas intracranianas, prevenção de inflamações pós-operatórias, restauração do contorno frontal e simetria ${ }^{4,2,9}$ e separação do trato nasossinusal e cérebro ${ }^{2}$. Diversos acessos às fraturas são descritos na literatura: incisão borboleta, incisão de Lynch, incisão em testallinha fina, lacerações, acesso coronal ou ainda via endoscópica? ${ }^{2}$. Todos pacientes tratados cirurgicamente em neste serviço foram submetidos a acesso coronal uma vez que se entende ser o mais estético para este tipo de fratura. A escolha do tratamento conservador deve ser tomada em consenso entre o cirurgião buco-maxilo-facial e o paciente e realizada com cautela. Devese observar se as funções desempenhadas pelo seio frontal estão em normalidade e se as alterações cosméticas são importantes ${ }^{11}$. Todos pacientes tratados cirurgicamente em neste serviço foram submetidos a acesso coronal uma vez que se entende ser o mais estético para este tipo de fratura, embora seja uma incisão extensa e comprometimentos estéticos possam surgir em pacientes com alopécia e haja o risco de lesão do ramo frontal do nervo facial (VII par) ${ }^{5}$.

As fraturas da tábua anterior do seio frontal ocorrem isoladamente em $43 \%$ dos casos, fraturas isoladas da tábua posterior em 0,6 a $6 \%$, fraturas da tábua anterior e posterior em 19 a $51 \%$ e envolvimento do ducto nasofrontal em 2,5 a $21 \%$ dos casos ${ }^{4}$. Em relação ao tratamento, não há consenso sobre quando a intervenção cirúrgica é indicada ${ }^{6}$.

Neste estudo, 38 pacientes $(74,5 \%)$ foram diagnosticados com fratura isolada da parede anterior do seio frontal e em 13 casos $(25,49 \%)$ a parede posterior também estava comprometida; destes, em 9 casos houve extravasamento de liquor celaforraquidiano (LCR) $\quad(17,64 \%) . \quad 37,25 \% \quad$ (19 pacientes) sofreram tratamento cirúrgico, em 1 caso o ducto nasofrontal encontrava-se obstruído unilateralmente, sendo necessário vedamento com osso. Optou-se por não se realizar a obliteração do seio frontal porque o ducto contralateral era evidente. Nenhum paciente foi submetido à cranialização do seio frontal por conduta da equipe de Neurocirurgia que acompanhava cada caso. O ducto nasofrontal localiza-se no soalho póstero-medial do seio frontal, mede de poucos milímetros até $1 \mathrm{~cm}$ ou mais e é responsável pela drenagem da mucosa do seio frontal até o meato nasal médio ${ }^{2,3}$. É chamado de "ducto verdadeiro" em $15 \%$ da população e em $80 \%$ a $85 \%$ dos casos, encontra-se ausente ${ }^{4}$. Fraturas associadas do tipo NOE, margem supra-orbitária e soalho do seio frontal devem chamar a atenção para a patência do ducto nasofrontal uma vez que esta é a chave para o tratamento ${ }^{4,5,7}$. A avaliação aberta ou intraoperatória é indicada, pois evidências sobre a confiabilidade na detecção 
do ducto nasofrontal em exames de tomografia computadorizada é escassa ${ }^{5}$. O muco do seio frontal é acumulado quando o ducto nasofrontal é destruído ou obstruído, com isso há aumento da atividade de bactérias anaeróbias e aumento do risco de sinusites ou cistos de cistos mucosos, por exemplo ${ }^{4,11}$. Se patente, permite saída de mucina, seroma ou hematoma após fratura. Se o ducto não é patente a remoção completa da mucosa remanescente do seio é realizada com a finalidade de isolar o seio frontal ou cérebro da contaminação nasal ${ }^{5,11}$. Qualquer remanescente da mucosa do ducto nasofrontal é invertida para o nariz $z^{5,9,11}$.

A obliteração do seio frontal tem como finalidade isolar este dos contaminantes nasais e eliminar espaço morto ou ar. É realizada a remoção total da mucosa sinusal e o espaço do seio frontal é preenchido com diversos tipos de materiais: osso, fáscia do músculo temporal, músculo, gordura, esponja de fibrina, hidroxiapatita, cartilagem ${ }^{4,5,7}$. No entanto Bell (2009) observa que este não é um passo necessário e, em sua prática, não se remove a membrana do seio desde que a drenagem nasofrontal permaneça intacta, embora alguns pesquisadores têm defendido a remoção da membrana do seio sempre que este tenha sido violado. Na eliminação do seio frontal mantemse as paredes anterior e posterior e é realizada meticulosa remoção de toda a mucosa visível e do córtex interno da parede do seio com uso de brocas sob abundante irrigação e a permanente oclusão do ducto nasofrontal ${ }^{5,6,8}$.

Já a cranialização do seio frontal ocorre em casos de cominutação da tábua posterior, lesão penetrante, extravasamento LCR, dano dural extenso ou dano lobo frontal. Acontece em $16 \%$ dos casos de fratura da parede posterior do seio frontal e há consequente aumento tamanho fossa craniana anterior. A remoção da tábua posterior é feita com brocas esféricas diamantadas ou pinças Kerrison e o neurocirurgião deve evitar a região de seio sagital por conta do sangramento abundante. Ocorre a exposição da dura-máter ou cérebro e tecidos não viáveis são removidos e lacerações durais reparadas; o cérebro expande para o espaço morto extradural e a tábua anterior é reconstruída 5,7,8,9.

Fraturasdeseiofrontalocorrememestreita proximidade com estruturas importantes como cérebro, olhos e nariz. Assim, complicações como meningites, abscessos cerebrais, extravasamento de líquor, diplopia, distopia e infecções são devastadoras em pacientes com este diagnóstico ${ }^{1}$. Em nossa experiência apenas dois pacientes apresentam ptose da pálpebra superior, em ambos os casos houve o diagnóstico de fratura panfacial com inúmeros traumas associados e traumatismos cranianos importantes com diversas sequelas de origem do sistema nervoso central. O diagnóstico preciso e elaboração de correto plano de tratamento, juntamente com atendimento das demais especialidades que atuam na da face, são mandatários no sucesso do atendimento de pacientes diagnosticados com fraturas panfaciais que envolvam o seio frontal ${ }^{6}$.

\section{Conclusão}

Após realização deste estudo retrospectivo através de prontuários, obteve-se uma prevalência de fraturas na parede anterior do osso frontal na população estudada no gênero masculino, com idade média de 34,39 anos. A principal causa desse tipo de traumatismo foi acidente de motocicleta, seguido do automobilístico. Em associação à fratura do osso frontal houve prevalência de fratura nasal, seguida e fraturas tipo NOE. As complicações variaram de comprometimento de base do crânio a lesões neurológicas como parestesia e rinorréia. $O$ melhor meio diagnóstico preconizado foi a tomografia computadorizada cônica, sendo os pacientes tratados cirurgicamente, com acesso coronal, embora esse tratamento promova comprometimento 
estético e pode resultar também em alopecia e lesão do ramo frontal do nervo facial.

\section{REFERÊNCIAS}

01. Sivori II LA, Leeuw R, Morgan I, Cunningham LL, Jr. Complications of Frontal Sinus Fractures With Emphasis on Chronic Craniofacial Pain and Its Treatment: A Review of 43 Cases 2010. American Association of Oral and Maxillofacial Surgeons. J Oral Maxillofac Surg 2010; 68:2041-2046.

02. Doonquah L, Brown P, Mullings W. Management of Frontal Sinus Fractures. Oral Maxillofacial Surg Clin N Am. 2012; 24:265-274

03. Molendijk J, Van der Wal KGH, Koudstaal MJ. Surgical treatment of frontal sinus fractures: the simple percutaneous reduction. Int. J. Oral Maxillofac. Surg. 2012; 41: 1192-1194.

04. Fonseca RJ, Walter RV, Betts NJ, Barber HD. Oral and Maxillofacial Trauma. Saunders, Phildelphia, 2009.

05. Conci RA, Martins JRP, Tomazi FH, Sbardelotto BM, Sirena Neto L, oliveira GR. Tratamento Cirúrgico de fratura de seio frontal. Surgical Treatment of Frontal Sinus Fracture. Rev. Cir. Traumatol. Buco-Maxilo-Fac. 2012; (12) 31-36.
06. Santos MBP, Cavalieri I, Araujo MM, Vale DS, Breda Junior MA. Tratamento de Fratura do Seio Frontal seguido da Obliteração do Sistema de Drenagem: Relato de Caso. Revista Portuguesa de Estomatologia, Medicina Dentária e Cirurgia Maxilofacial.2010 (51)4.

07. Miloro M et al. Peterson's Principles of Oral and Maxillofacial Surgery. 2nd ed.B C Decker Inc, New York, 2009.

08. Bell RB. Management of Frontal Sinus Fractures. Oral Maxillofacial Surg Clin N Am. 2009; (21) 227-242.

09. Javer AR, Alandejani T. Prevention and Management of Complications in Frontal Sinus Surgery. Otolaryngol Clin N Am. 2010; (43) $827-83$

10. Ruggiero FP, Zender CA. Frontal sinus cranialization. Operative Techniques in Otolaryngology. 2010; (21) 143-146.

11. Carincia F, Zollinoa I, Arduina L, Lapparellib M, Cavallob M, Cenzic R. Fronto-ethmoidal fractures: A staging system and case series analysis. Asian Journal of Oral and Maxillofacial Surgery. 2010; (22) 74-79.

Submetido em: 1-6-2013

Aceito em: 11-11-2014 